\title{
NANOCOMPOSITES AND THEIR USE IN TECHNICAL PRACTICE
}

\author{
Jan Novotny, Martin Jaskevic \\ J. E. Purkyne University in Usti nad Labem, Czech Republic \\ novotny@fvtm.ujep.cz, martin.jaskevic@ujep.cz
}

\begin{abstract}
This paper describes the formation of a nanocomposite coating. It is a PTFE coating with the addition of TiAlV6 and WC (tungsten carbide) in size "nano". Working with nanoparticles requires not only safety, but also preparation of nano size particles and their subsequent uniform deployment (the particles of this size affect electrostatic attractive forces and tend to aggregate). The first part describes preparation of particles by the process of mechanical grinding, the second part deals with the particle concentrations in relation to the total content of the solution that have been used. Further, the coating technology is described, since dispersed particles in solution during coating are susceptible to settling at the bottom of the coating vessel. The Scanning Electron Microscope (SEM) and Energy Dispersive Spectrometry (EDS) were used to evaluate the particle size and particle size distribution on the sample surface.
\end{abstract}

Keywords: nanoparticles, WC, TiAlV6, coating, grinding processes, PTFE.

\section{Introduction}

The coating with TiAlV6 and WC will be used for molds used in vulcanization of rubber, especially for automotive tire production. The substrate for the coating is Al-Si type. The nanocomposite coating consists of PTFE coating and TiAlV6-based particles and WC. Research into the use of nanoparticles is currently developing very much. Their potential benefits greatly. Particles of nano size, i.e. less than $100 \mathrm{~nm}$, exhibit properties other than larger particles, and are therefore investigated in all possible sectors. The effect of adding these particles with different mechanical properties in coatings on different surfaces (separated on a band saw, milling, roughness 800 and 80 polished) depends on the quality of the prepared nanoparticles by the different grinding conditions. The aim is to find a suitable type of particles (alloy, oxide ...), the process of preparing these particles (grinding) and concentrating the coating solution to achieve a longer lifetime (abrasion resistance, thermal conductivity) after the application of the coating.

\section{Grinding processes}

We use the PM 100 planetary ball mill from Retsch, a $250 \mathrm{ml}$ mill of $\mathrm{ZrO} 2$ and a $3 \mathrm{~mm}$ diameter grinding sphere made of $\mathrm{ZrO} 2$. We have used the knowledge from the previous research (NOVOTNY, J., JASKEVIC, M. Morphology and producing of TiO2 particles, Engineering for Rural Development, 2016 Vol. 15, Latvia, pp. 1218-1223, 2016, ISSN 1691-5976) and have chosen the optimal input conditions for milling to obtain the finest grain fraction we require. Both samples were processed using the same procedure. The first grinding was done by the dry method and the second - wet milling method (colloid) in demineralized water. Input grinding conditions are summarized in Table 1.

Input grinding conditions

Table 1

\begin{tabular}{|l|c|l|c|}
\hline \multicolumn{4}{|c|}{ Material: TiAlV6 a WC } \\
\hline Weight & $100 \mathrm{~g}$ & Weight & $2{ }^{\text {nd }}$ wet grinding \\
\hline Total grinding time & $60 \mathrm{~min}$ & Total grinding time & $2000 \mathrm{~min}$ \\
\hline Speed & $250 \mathrm{rpm}$ & Speed & $300 \mathrm{rpm}$ \\
\hline Grinding interval & $5 \mathrm{~min}$ & Grinding interval & $10 \mathrm{~min}$ \\
\hline Break interval & $55 \mathrm{~min}$ & Break interval & $50 \mathrm{~min}$ \\
\hline Grinding medium & air & Grinding medium & DEMI water \\
\hline Vol. of grinding medium & - & Vol. of grinding medium & $100 \mathrm{ml}$ \\
\hline BPR & $5: 1$ & BPR & $17: 1$ \\
\hline
\end{tabular}

After each grinding, a sample of the obtained particles was collected and analyzed by a scanning electron microscope. The grain morphology can be seen in Figures 1-4. 


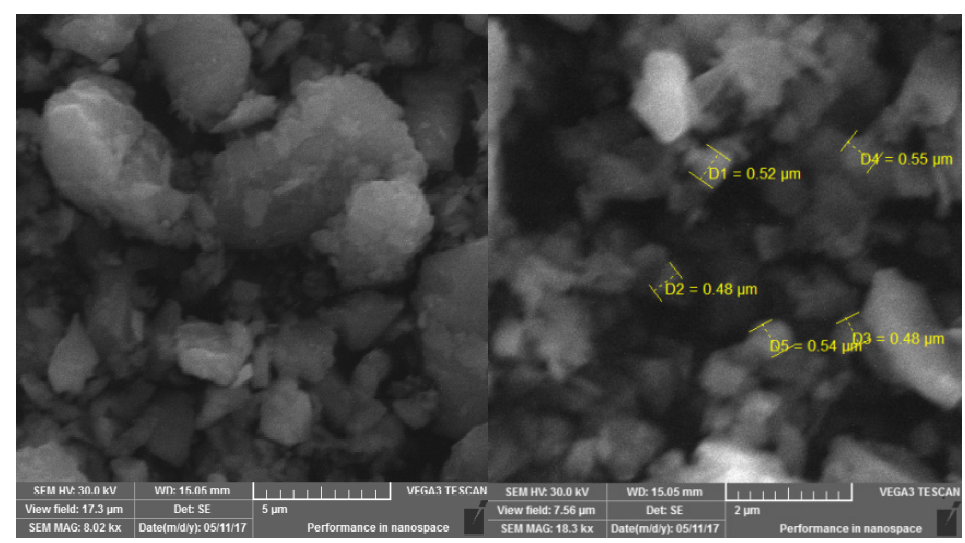

Fig. 1: TiAlV6 dry grinding

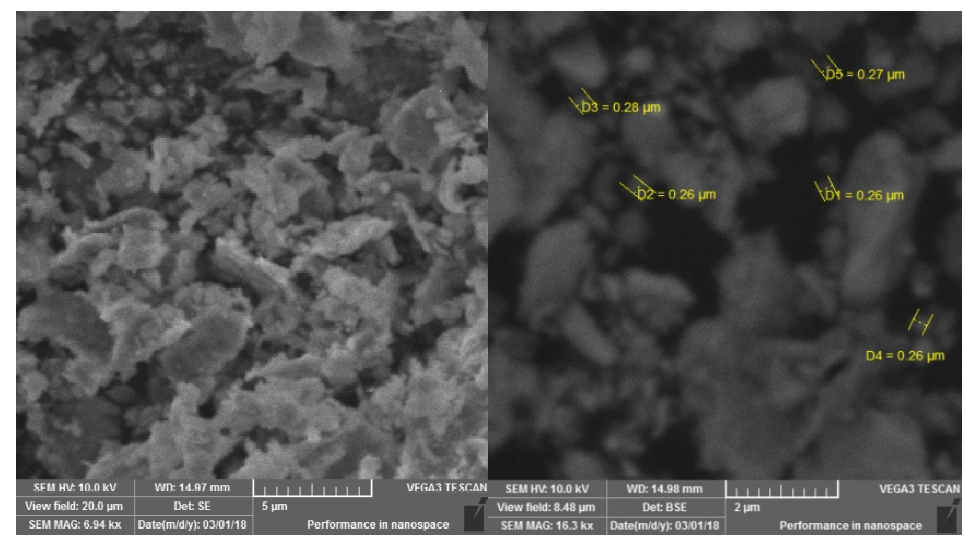

Fig. 2: TiAlV6 wet grinding

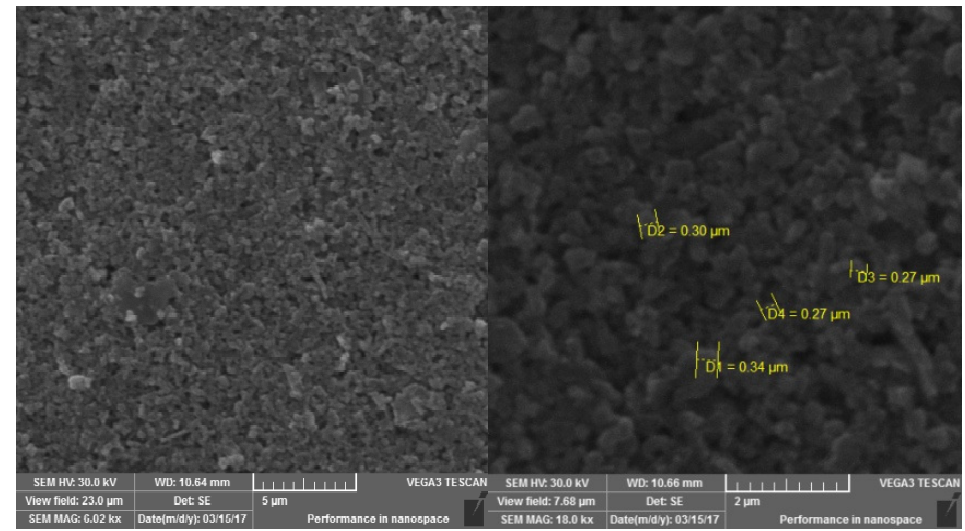

Fig. 3: WC dry grinding

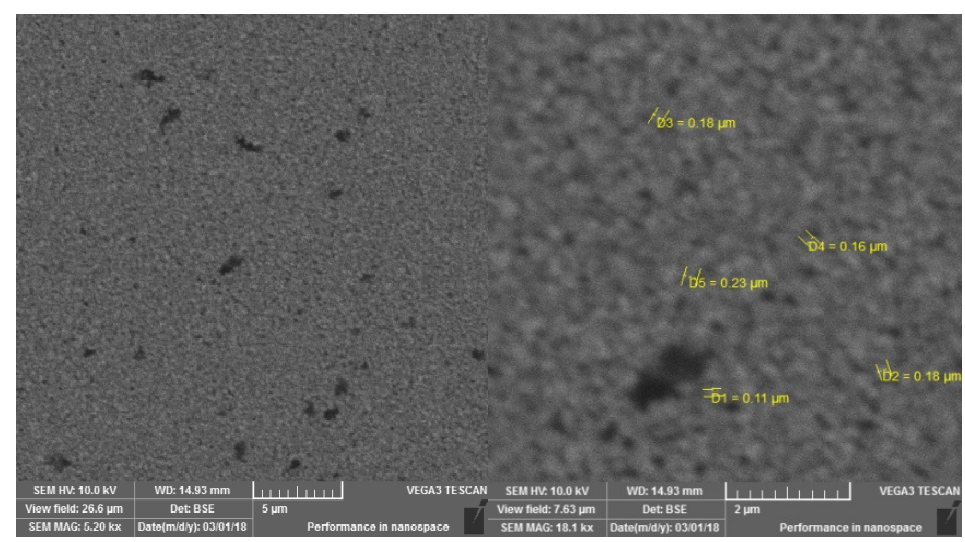

Fig. 4: WC wet grinding 
Figure 1 is TiAlV6 after the first dry grinding. The grain is sharp and irregular. In larger numbers there is also a coarser fraction. After the second colloidal grinding (Figure 2), further grain size reduction and especially homogenization of the particle size distribution are visible and no larger particles are present. For the WC in Figures 3 and 4 we observe a similar trend as in the previous case and the homogenization of the particle size is very good.

\section{Concentration of coating solutions and coating}

The coating solution consists of demineralised water, Teflon emulsion and particulates. The coating process takes place according to the patented pattern that is used for coating $\mathrm{Al}$ - Si forms. For the final coating layer, we chose ALFIFLON 39 PTFE emulsion. The resulting surface properties influence both the composition of the particles used and the particle concentration in the coating solution and thus on the surface of the coated sample. For research, we chose three sizes of concentrations for both materials:

- $0.1 \%$

- $0.01 \%$

- $0.001 \%$.

The coating process consists of dipping the samples into several solutions of the given composition and temperature, and subsequent drying. In the final phase - in solution with scattered particles. Because of the small particle size aggregation occurs here and particles need not form clumps and can freely dissolve in the solution. For this purpose an ultrasonic cleaner is used in the pretreatment phase, which destroys the resulting aggregation of the particles. Another problem arises during the coating itself, when the material particles tend to drop to the bottom of the vessel due to their higher density and become sedimentary. This phenomenon is evident especially at higher concentrations. To prevent sedimentation and uniform dispersion of particles in the solution, a magnetic stirrer is used to ensure a homogeneous distribution of the particles in the solution.

\section{SEM a EDS sample analysis}

The resulting sample surface was again subjected to the SEM analysis and subsequent EDS analysis. To verify the suitable concentrations a scanning electron microscope TESCAN Vega 3 EDS analyzer equipped with a Bruker X-FLASH was used. Figure 5 shows a $0.1 \%$ TiAlV6 coating.

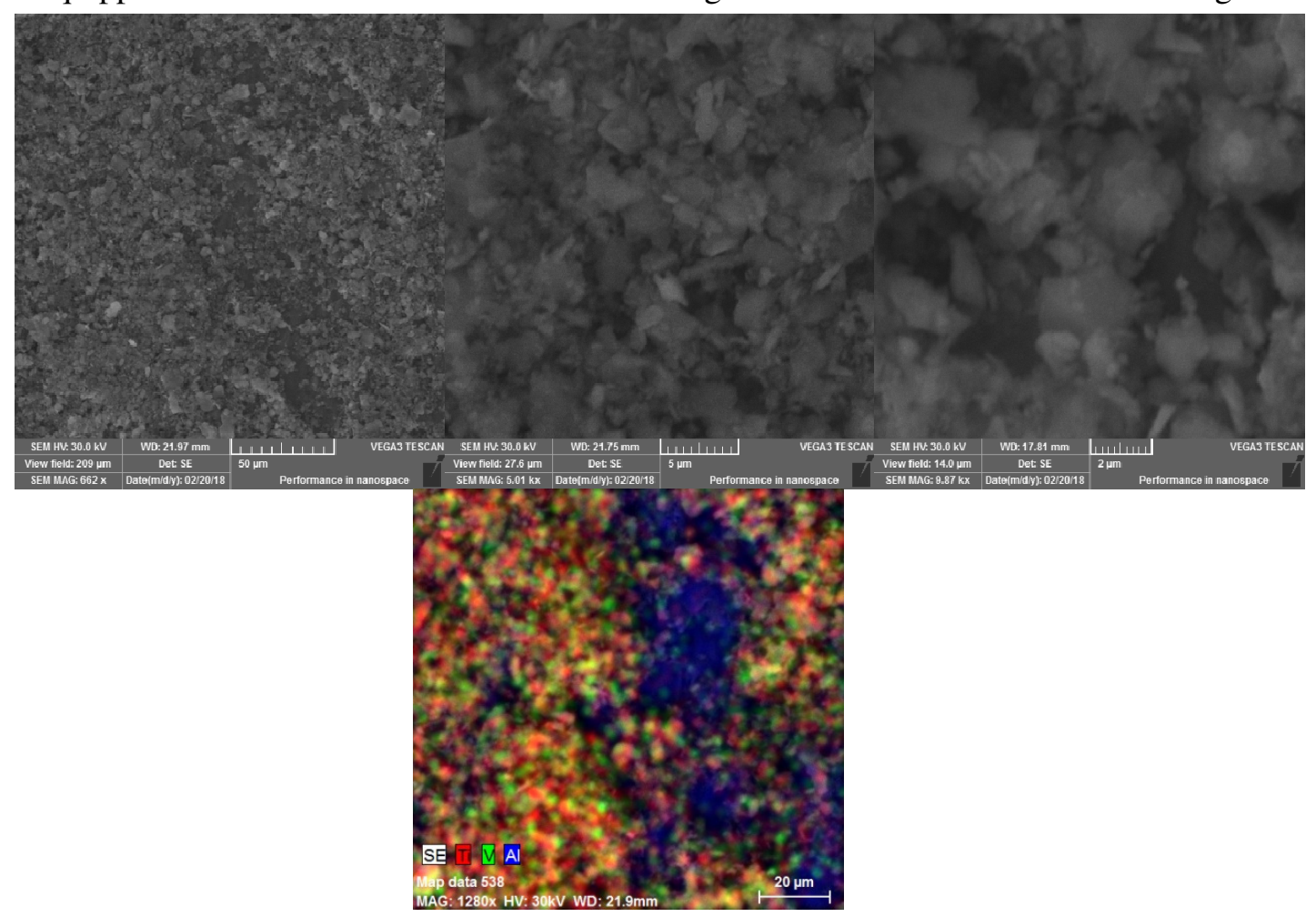

Fig. 5. TiAlV6 0.1 \% solution 
We can see that the surface of the sample is covered with particles at this high concentration. High concentrations may lead to incomplete adhesion of the particles to the surface and wiping of particles by abrasion. From the EDS analysis, it is clear that the particles are scattered on the surface horizontally.

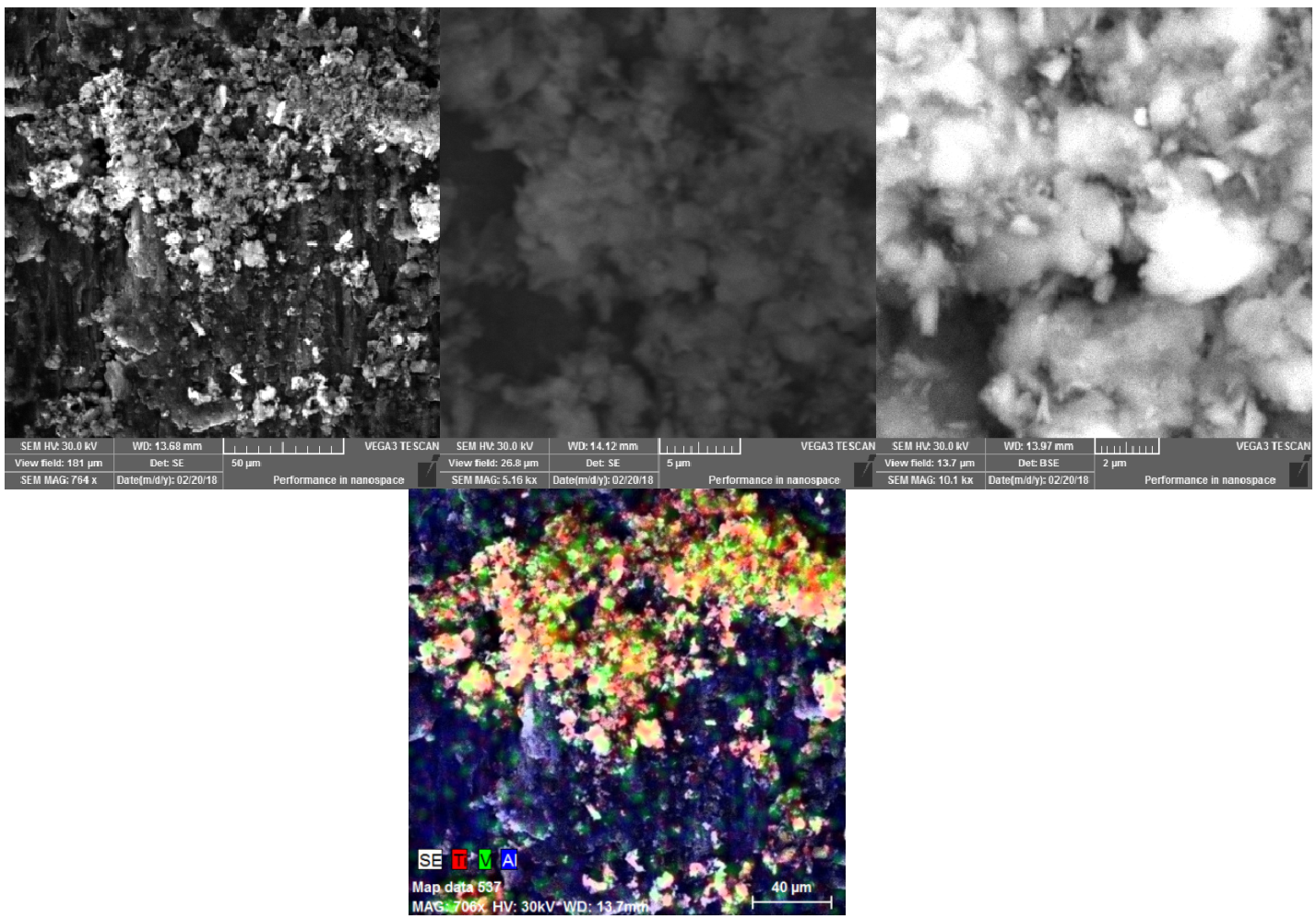

Fig. 6. TiAlV6 0.01 \% solution

Fig. 6 shows a $0.01 \%$ coating. It is evident that the concentration is no longer sufficient to cover the entire surface of the component and places with a higher particle concentration on the surface.

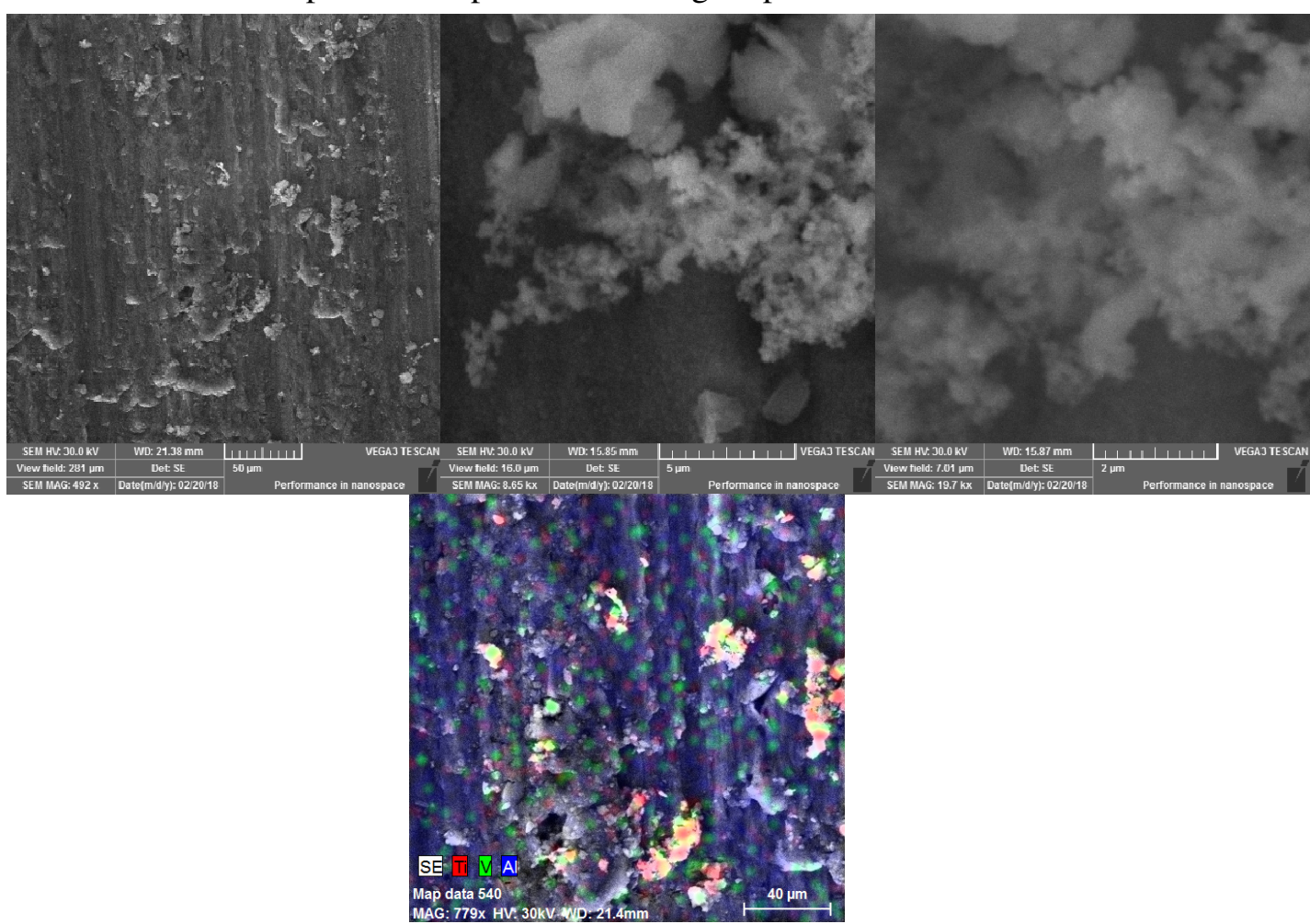

Fig. 7. TiAlV6 0.001 \% solution 
In the $0.001 \%$ of the coating of Figure 7, this phenomenon is even more apparent. The particles in the solution are no longer enough to cover most of the surface and make particles free of particles.

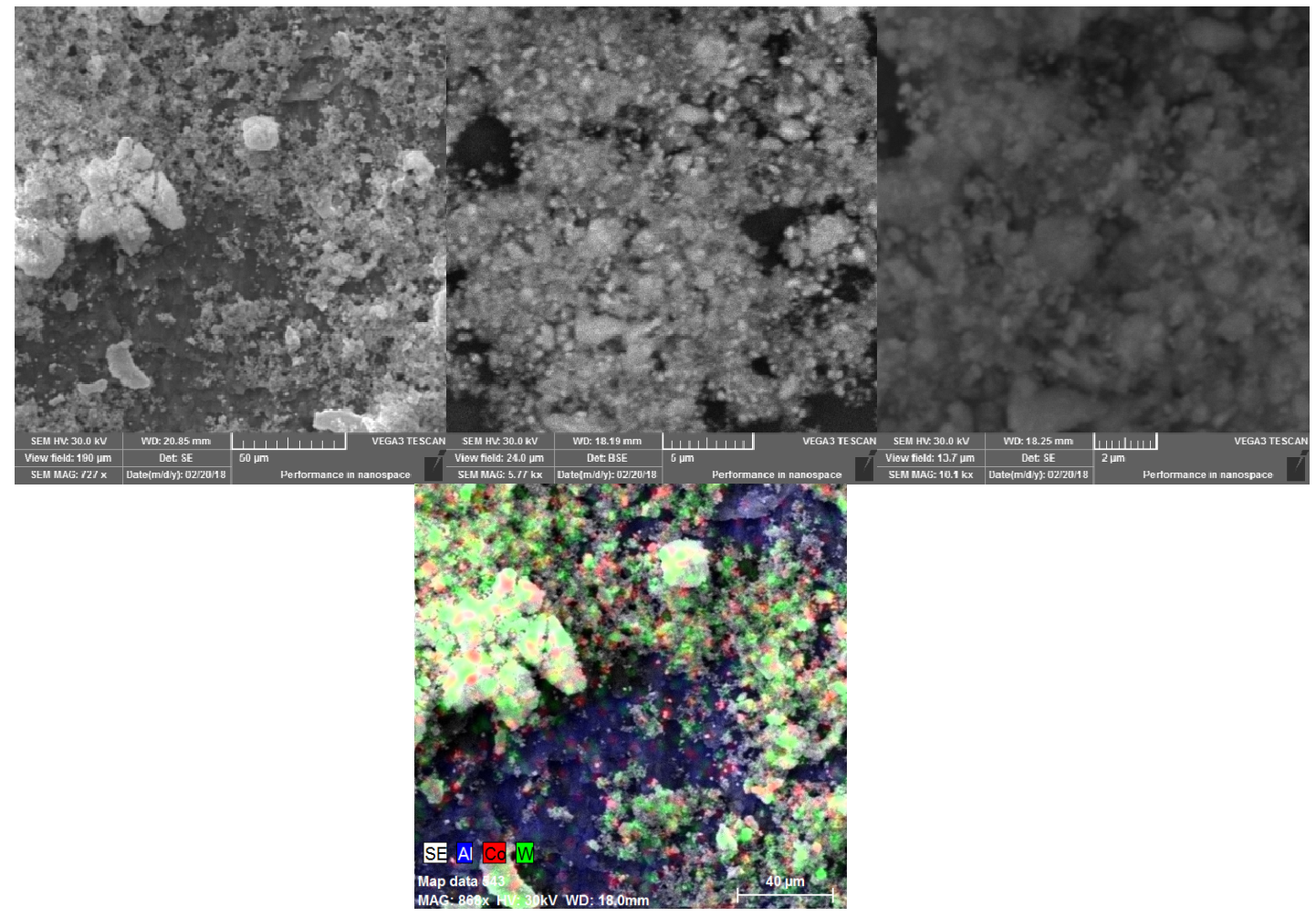

Fig. 8. WC $0.1 \%$ solution

As in the previous case, for concentrating $0.1 \% \mathrm{w} / \mathrm{w}$ solution, the entire surface of the sample is covered with particles as shown in Figure 8.

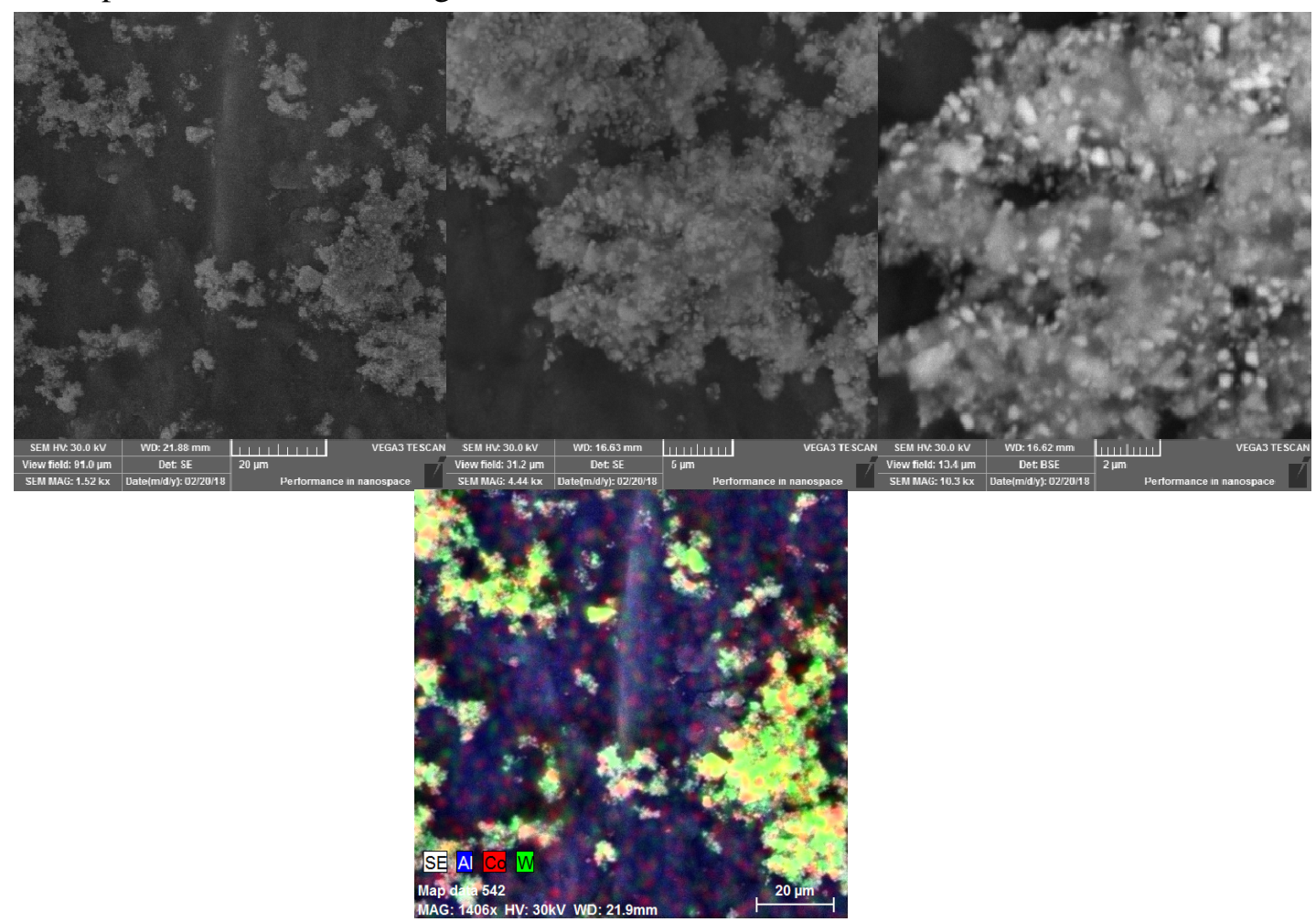

Fig. 9. WC 0.01\% solution

At a concentration of $0.01 \% \mathrm{WC}$ we see in Figure 9 that the surface coverage of the particles is lower than that of the TiAlV6 concentration. 

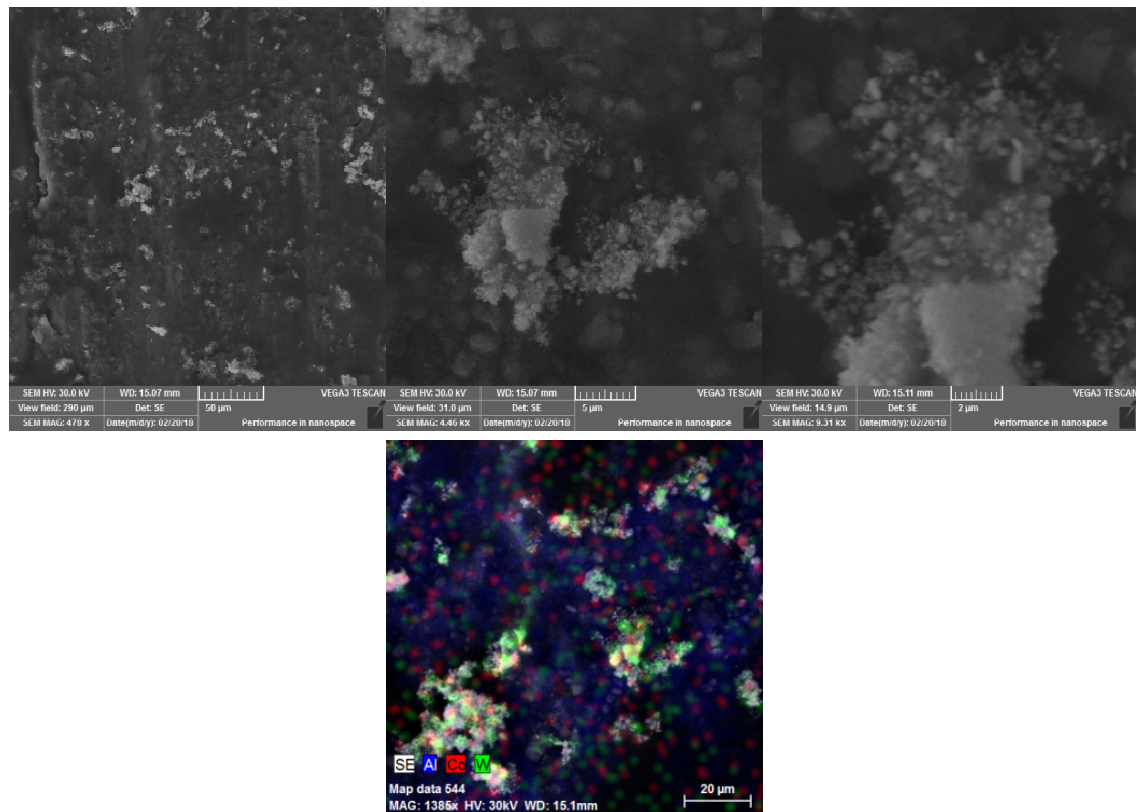

Fig. 10. WC 0.001 \% solution

The lowest concentration confirms this trend and most of the surface remains free of particles, as shown in Figure 10.

\section{Conclusions}

The first part describes the TiAlV6 and WC process. This is mainly the setting of the ball mill and the entry conditions of grinding. Dry milling was chosen as sample preparation for subsequent colloidal grinding in demineralized water. As it can be seen, after 60 minutes of dry grinding, the fraction is substantially refined. After 2000 minutes of colloidal grinding, there is a further reduction in the size of the fraction, but the refining is no longer proportional to the grinding time. In particular, the particle size distribution is homogenized towards smaller sizes, which is advantageous. The next section describes the process of coating the samples and, above all, solving the problems associated with coating, such as breaking aggregates and dispersing the particles in the coating solution. In the last part, the SEM and EDS analyses in the images are captured by the surface and composition of the coated samples. It can be seen that with the decreasing concentration of the solution, the percentage of particle surface coverage of the component decreases. On the contrary, this may have a positive effect on the resulting surface properties, because at higher concentrations there may be particle abrasion just because of a strong particle layer on the surface.

\section{References}

[1] Bhushan B. Springer handbook of nanotechnology, Springer, 2003. ISBN 978-3-642-02525

[2] Giacovazzo C. Fundamentals of Crystallography, Oxford University Press, USA 2002.

[3] Michna Š., Cais J. CZ/28.07.15/CZA 20150521 Aluminium alloy in particular for the production of mould segment castings for forming types and the method of heat treatment of mould segment castings

[4] Novotný J., Lysonkova I., Naprstkova N., Michna S. Research of application possibilities of selected mechanically alloyed metal powders, In Manufacturing Technology, Vol. 17 No. 5, FSI UJEP, Ústí nad Labem, 2017, pp. 811-815.

[5] Novotny J., Jaskevic M. Morphology and producing of TiO2 particles, Engineering for Rural Development, 2016 Vol. 15, Latvia, 2016, pp. 1218-1223,

[6] Novotný J., Jaskevič M., Cais J. Hung T. D. The shape and structure of titanium dioxide nanoparticles, In Manufacturing Technology, Vol. 16, No. 5, FVTM UJEP, Ústí nad Labem, 2016, pp. 1102-1106. 
[7] Lysonkova I., Novotny J., Cais J., Michna S. Extended of Durability Molds for Production Tires with New Types of Coatings. Proceedings of the International Conference on Science \& Engineering for Sustainable Development, 2017, pp. 358-363. doi: http://dx.doi.org/10.21013/jte.ICSESD201734

[8] Lysonkova I., Novotny J., Cais J., Michna S. Effect of addition of nanoparticles $\mathrm{TiO}_{2}$ into PTFE coating, In Engineering for Rural Development, 2017 Vol. 16, Latvia, 2017, pp. 26-30.

[9] Müller M. Effects of Aluminium Microparticles and Surface Treatment of AlCu4Mg on Mechanical Properties of Adhesive Bond Strength, In Manufacturing Technology, Vol. 17, No. 1, FVTM UJEP, Ústí nad Labem, pp. 66-71.

[10] Valašek P., Muller M. Polymeric particle composites with filler saturated matrix. Manufacturing Technology, Ústí nad Labem 2012 Voume 12. ISSN 1213-2489 